\title{
Preparation of Silver Nanoparticles by Arc Plasma Method and Their Properties*1
}

\author{
Takahiro Mineta, Tatsuya Saito*2, Takahiro Yoshihara*2,*3 and Hiroyuki Sato \\ Department of Mechanical Science and Engineering, Graduate School of Science and Technology, \\ Hirosaki University, Hirosaki 036-8561, Japan
}

\begin{abstract}
Ag nanoparticles were prepared by the arc plasma method under various conditions. The particle properties, such as size, size distribution, shape, and purity, were investigated. It was revealed that the average size of the Ag nanoparticles decreased with increasing arc current during the application of the arc plasma method. Moreover, the crystallite size measured using X-ray diffraction (XRD) was smaller than the average particle size, regardless of the arc current. Thus, it was concluded that the Ag nanoparticles prepared by this method are polycrystalline particles. No Ag oxides were observed in the nanoparticles by either field emission scanning electron microscopy observations or XRD analysis. Moreover, the solid solution of oxygen in Ag was not detected by XRD or wavelength dispersive X-ray spectrometry analysis. [doi:10.2320/matertrans.M2018391]
\end{abstract}

(Received December 21, 2018; Accepted January 29, 2019; Published March 25, 2019)

Keywords: silver, arc plasma method, nanoparticles, crystallite, Halder-Wagner method, particle size distribution

\section{Introduction}

Ag nanoparticles, which are smaller than $1 \mu \mathrm{m}$, are attracting attention for application in various industrial fields, such as the electronics industry. Recently, there has been increasing demands for Ag nanoparticles with various controlled particle properties, such as size, size distribution, shape, and purity, to achieve the miniaturization of electronics. Moreover, sintered materials prepared using nanoparticles are expected to show excellent strength, because strength increases with decreasing grain size. ${ }^{1,2)} \mathrm{Ag}$ shows good biocompatibility and has been applied as a biomedical material; however, Ag strengthening by the addition of alloying elements is necessary because of its low strength. ${ }^{3,4)}$ The alloying elements cause the degradation of its biocompatibility. Thus, it is preferable to increase the strength of $\mathrm{Ag}$ without the addition of alloying elements for biomedical applications, and sintering using Ag nanoparticles can be done for this purpose. Since the grain size and mechanical properties of $\mathrm{Ag}$ are affected by the properties of the nanoparticles, control of the particle properties is necessary in the preparation of a high-strength sinter of Ag.

Various methods for preparing $\mathrm{Ag}$ particles, such as milling, ${ }^{5)}$ atomization, ${ }^{6,7)}$ and chemical reduction processes, ${ }^{8)}$ have been proposed. However, these methods have various challenges, such as the preparation of nanoparticles, avoidance of contamination by impurities, and minimization of energy consumption for the preparation of nanoparticles. In this study, the arc plasma method was selected for the preparation of Ag nanoparticles. With this method it is easier to prepare uniform nanoparticles of various materials without contamination by impurities in comparison to other methods. ${ }^{9-15)}$ Thus, preparation of Ag nanoparticles by the arc plasma method is considered suitable for industrial applications. However, the relationship between the preparation conditions of Ag nanoparticles and the particle properties

\footnotetext{
${ }^{* 1}$ This Paper was Originally Published in Japanese in J. Japan Inst. Met. Mater. 83 (2019) doi:10.2320/jinstmet.JBW201801.

${ }^{* 2}$ Graduate Student, Hirosaki University

*3Present address: Panasonic Corporation, Osaka 571-7502, Japan
}

in the arc plasma method is insufficiently understood because this method has only been reported in few previous studies. ${ }^{16)}$ Moreover, nanoparticles generally have a significantly large surface area per unit volume. Thus, the oxidation of nanoparticles rapidly progresses at the particle surfaces, resulting in changes in their chemical composition. ${ }^{17,18)}$ Hence, it is valuable to explore the oxidation behavior of Ag nanoparticles prepared by the arc plasma method.

In this study, Ag nanoparticles were prepared by the arc plasma method, and the influence of the magnitude of the arc current on the nanoparticle properties was investigated. It has been previously reported that the arc current during application of the arc plasma method significantly affects the nanoparticle properties. ${ }^{19-21)}$ Thus, the properties of Ag nanoparticles prepared with various arc currents were investigated. The size, the size distribution, shape, and purity were selected as the particle properties for investigation, and they were quantitatively assessed by various methods.

\section{Experimental Procedure}

\subsection{Preparation of Ag nanoparticles by arc plasma method}

A schematic of the nanoparticle preparation apparatus for the arc plasma method is shown in Fig. 1. In this study, an arc melting furnace equipped with a gas circulator and a nanoparticle collecting filter was used (ATTOTEC Co., Ltd., UFP-300). High-purity Ag (>99.9\%, oxygen content: $70 \mathrm{ppm}$ ) was placed on a water-cooled high-purity graphite hearth. After a base vacuum was achieved, the apparatus was filled with Ar gas, then a base vacuum was achieved again. Then, the apparatus was filled with $\mathrm{Ar}-50 \% \mathrm{H}_{2}$ mixture gas. The arc plasma was generated with a gas flow rate of $50 \mathrm{~L} \cdot \mathrm{min}^{-1}$, and the prepared Ag nanoparticles were subsequently transported and collected by the filter in the collector chamber. The arc current values were 40, 50, and $80 \mathrm{~A}$.

\subsection{Characterization of Ag nanoparticles}

For examination of the crystallographic structure, the lattice constant, and the crystallite size of the Ag nano- 


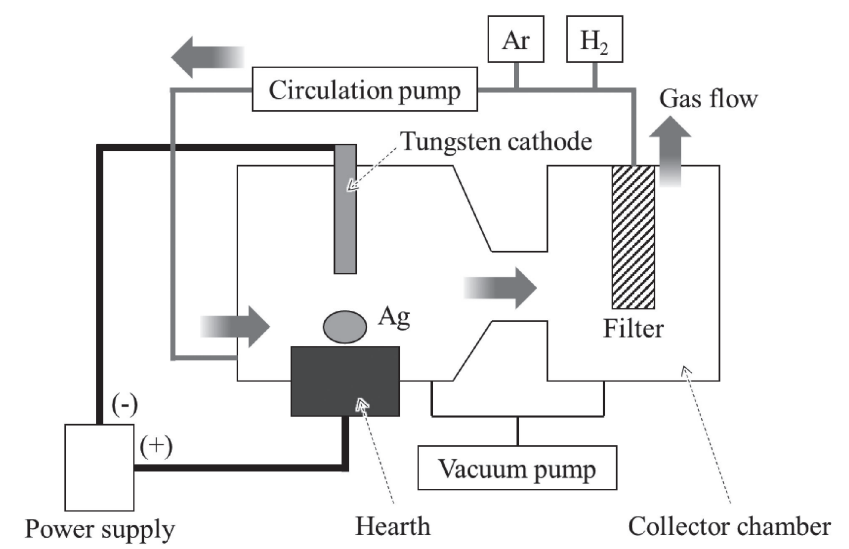

Fig. 1 Schematic of the equipment for the arc plasma method used to prepare Ag nanoparticles.

particles, X-Ray diffraction (XRD: Rigaku, SmartLab $9 \mathrm{~kW}$ ) analysis was conducted. XRD was selected for the analysis of the crystallite size because the average crystallite size of a larger number of particles can be measured quantitatively by this method than by transmission electron microscopy. We collected XRD data using $\mathrm{Cu}-\mathrm{K} \alpha$ radiation (wave length: $\lambda=0.15406 \mathrm{~nm}$ ) with $40 \mathrm{kV}$ and $200 \mathrm{~mA}$ over a $2 \theta$ range of $30^{\circ}$ to $80^{\circ}$ and a step size of $0.01^{\circ}$ at room temperature. The XRD analysis was conducted using the Rigaku, PDXL XRD analysis software version 2.7.3.0. The pseudo-Voigt function was used for XRD profile fitting. The crystallite size $D$ of each sample of $\mathrm{Ag}$ nanoparticles was evaluated using the (111), (200), (220), (311), (222), and (400) diffraction peaks of Ag with the face-centered cubic (FCC) structure by the Halder-Wagner ${ }^{22)}$ and Scherrer methods. ${ }^{23}$ ) Wavelength dispersive X-ray spectrometry (WDS: JEOL, JXA-8230) was conducted for quantitative analysis of the oxygen content.

The morphology of the Ag nanoparticles was investigated by field emission scanning electron microscopy (FE-SEM: JEOL, JSM-7000F), and the particle size distributions were clarified by an image analysis using software (ImageJ ver. 1.451). The particle size analysis was performed using more than 300 nanoparticles.

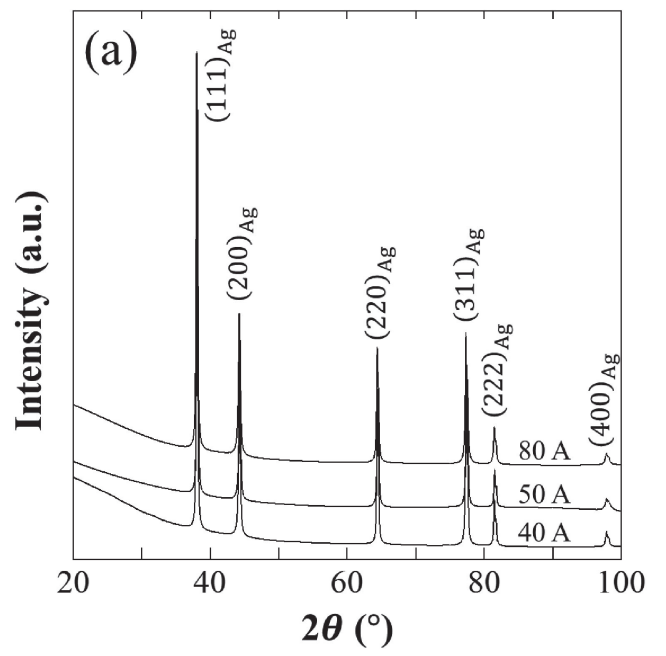

\section{Results and Discussion}

\subsection{Crystallographic structure and oxygen content}

Figure 2(a) shows the XRD patterns of each Ag nanoparticle sample. As seen in this figure, it was confirmed that all of the Ag nanoparticles consisted of a single FCC phase, and Ag oxide was not detected. The lattice constant of the Ag nanoparticles measured in this study was $0.4086 \mathrm{~nm}$. This value is good agreement with the reported lattice constant of $\mathrm{Ag}(0.4086 \mathrm{~nm}) .{ }^{24)}$ Note that it has been previously pointed out that the oxygen solute atoms in Ag cause changes in the lattice constant. ${ }^{25,26)}$ Since the lattice constant of the $\mathrm{Ag}$ nanoparticles measured by XRD agrees with the reported value, it is concluded that the amount of solute oxygen in the nanoparticles was negligibly small. In addition, the WDS analysis results show that the oxygen content in the $\mathrm{Ag}$ nanoparticles was below the detection limit (100 ppm), and no Ag oxides were found by FE-SEM. Therefore, it is concluded that the oxygen content does not change during the nanoparticle preparation process, and Ag nanoparticles with negligibly small oxygen content can be prepared by the arc plasma method proposed in this paper.

\subsection{Crystallite size}

The crystallite size of each Ag nanoparticle was evaluated by examination of the XRD patterns shown in Fig. 2. According to the Halder-Wagner method, the crystallite size $D$ can be expressed as

$$
\begin{aligned}
\left(\frac{\beta^{*}}{d^{*}}\right)^{2} & =\frac{1}{D} \frac{\beta^{*}}{\left(d^{*}\right)^{2}}+\left(\frac{\varepsilon}{2}\right)^{2}, \\
\beta^{*} & =\beta \cos \theta / \lambda, \\
d^{*} & =2 \sin \theta / \lambda,
\end{aligned}
$$

where $\beta$ is the integral breadth, $\theta$ is the Bragg angle, and $\varepsilon$ is the microstrain. According to eq. (1), the inverse of the slope of the Halder-Wagner plot $\left(\left(\beta^{*} / d^{*}\right)^{2}\right.$ versus $\left.\beta^{*} /\left(d^{*}\right)^{2}\right)$ gives the crystallite size $D$. Figure 3 shows the Halder-Wagner plots and straight lines fitted by the least-squares method of each Ag nanoparticle. The crystallite sizes $D$ of the Ag nanoparticles prepared by the arc plasma method with the arc

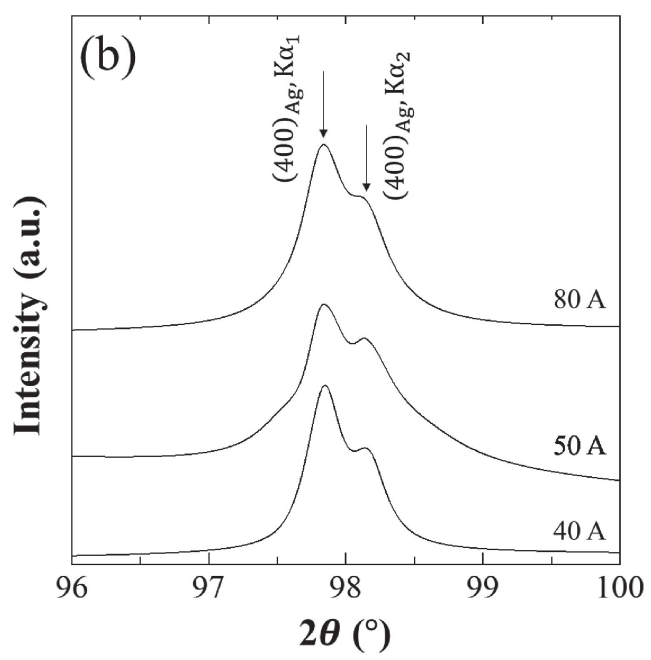

Fig. 2 XRD patterns of each Ag nanoparticle sample. 


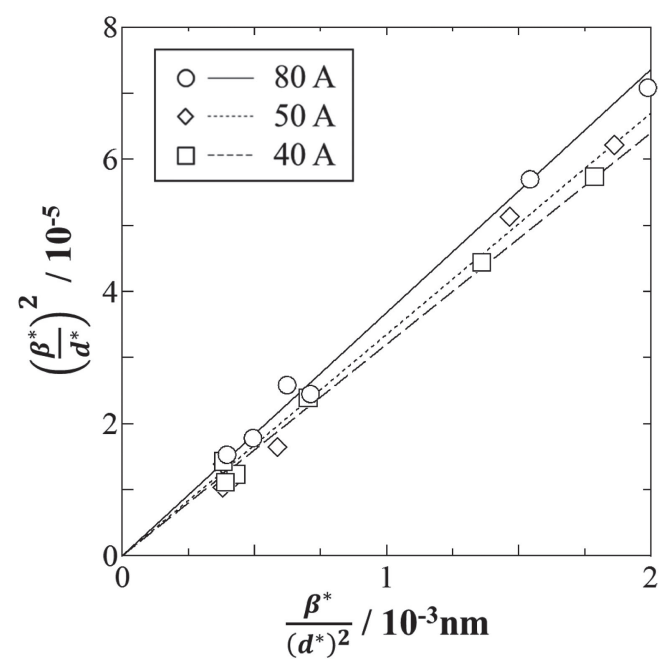

Fig. 3 Halder-Wagner plots for each Ag nanoparticle sample.

currents of 40,50 , and $80 \mathrm{~A}$ were $D_{40 \mathrm{~A}}=30.3 \mathrm{~nm}, D_{50 \mathrm{~A}}=$ $29.2 \mathrm{~nm}, D_{80 \mathrm{~A}}=27.1 \mathrm{~nm}$, respectively. Thus, $D$ decreased with increasing arc current value; however, the change in the crystallite size $D$ was small. A magnified image of the XRD patterns is shown in Fig. 2(b). No obvious difference in the integral breadth of each (400) diffraction peak was observed, although the integral breadth generally increased with decreasing crystallite size. The crystallite sizes $D^{\prime}$ evaluated by the Scherrer method for the Ag nanoparticle samples were $D_{40 \mathrm{~A}}^{\prime}=23.8 \mathrm{~nm}, \quad D_{50 \mathrm{~A}}^{\prime}=24.8 \mathrm{~nm}$, and $D_{80 \mathrm{~A}}^{\prime}=22.5 \mathrm{~nm}$. These values are consistent with those evaluated by the Halder-Wagner method. Thus, it is concluded that the values of the crystallite size evaluated in this study are reasonable. The increase in the arc current corresponds to the increase in energy applied to the material during arc discharge; however, the crystallite size $D$ does not depend on the arc current.

\subsection{Particle size, size distribution, and shape}

FE-SEM images of each Ag samples are shown in Fig. 4. As seen in this figure, spherical Ag nanoparticles can be prepared by the arc plasma method. It is also clear that some nanoparticles aggregated during operations. The particle size distribution and standard deviation (SD) of each $\mathrm{Ag}$ nanoparticle sample are shown in Fig. 5. The average particle size $\bar{d}$ and the crystallite size of each Ag sample are shown in Fig. 6. The particle size of the $\mathrm{Ag}$ nanoparticles was calculated from the projected area of each nanoparticle measured by image analysis using ImageJ software because the shape of nanoparticle was spherical. Note that the particle size of above-mentioned aggregated Ag nanoparticles was calculated by estimating the spherical projection area before aggregation. The results revealed that the average particle size and the SD decreased with increasing arc current. In other words, an increase in the energy applied to the raw material during arc discharge leads decreased particle size and SD. Among the Ag nanoparticle preparation conditions in this study, the $\mathrm{Ag}$ nanoparticles prepared with the arc current of $80 \mathrm{~A}$ showed the smallest average particle size $(\bar{d}=86.0 \mathrm{~nm})$, the smallest median particle size $\left(d_{50}=\right.$ $79.3 \mathrm{~nm}$ ), and the smallest SD of the particle size distribution. Farbod et al. investigated the relationship between the particle size of various materials and the arc current during the application of the arc plasma method. They reported that the particle size decreased with increasing arc current under some conditions. ${ }^{19-21)}$ Moreover, their studies indicated that the influence of the arc current on the average particle size may depend on the gas atmosphere during application of the arc plasma method. Although the reason why the average particle size of Ag nanoparticles depends on the arc current is unclear in this study, it is suggested that further miniaturization of $\mathrm{Ag}$ nanoparticles can be attained by controlling the particle preparation conditions, such as the arc
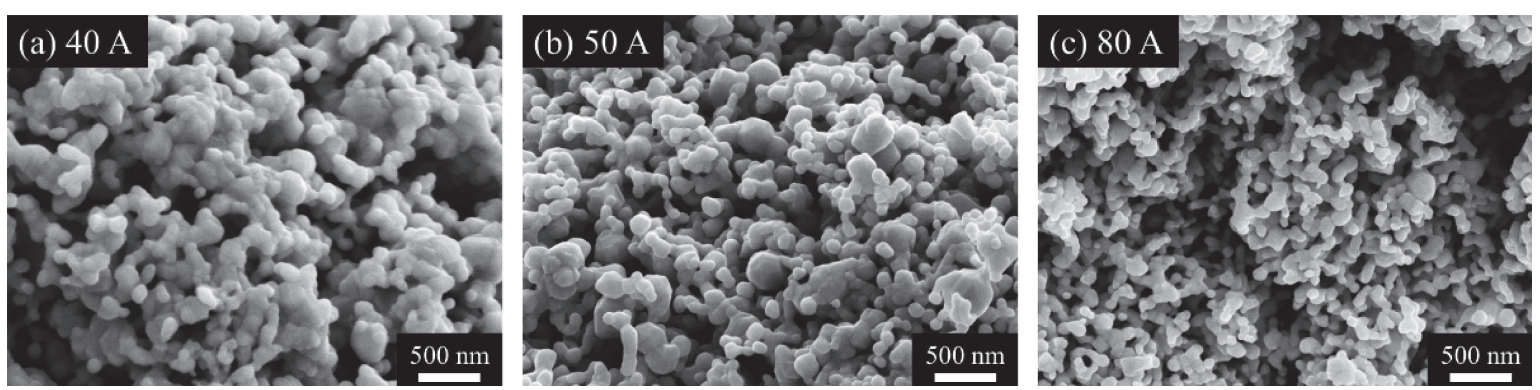

Fig. 4 FE-SEM images of Ag nanoparticles prepared by the arc plasma method with arc currents of (a) 40, (b) 50, and (c) 80 A.

(a) $40 \mathrm{~A}\left(\mathrm{SD}=36.9 \mathrm{~nm}, d_{50}=123.3 \mathrm{~nm}\right)$

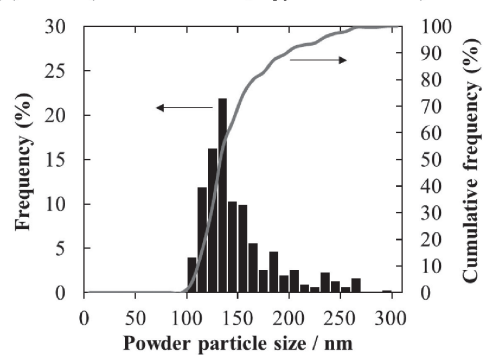

(b) $50 \mathrm{~A}\left(\mathrm{SD}=30.3 \mathrm{~nm}, d_{50}=106.4 \mathrm{~nm}\right)$

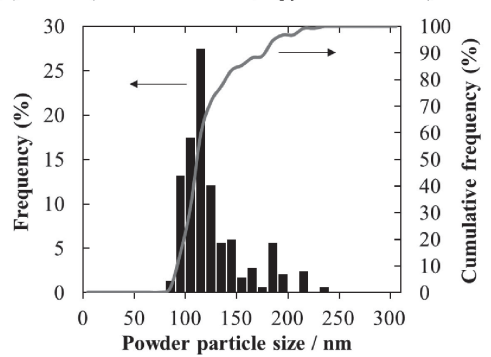

(c) $80 \mathrm{~A}\left(\mathrm{SD}=19.7 \mathrm{~nm}, d_{50}=79.3 \mathrm{~nm}\right)$

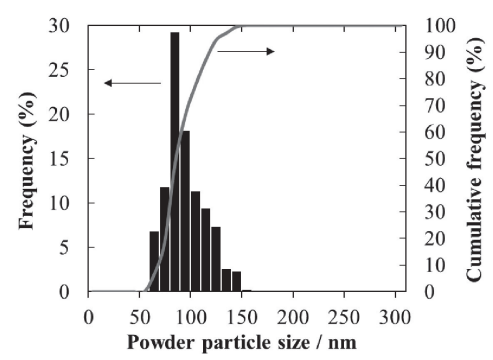

Fig. 5 Powder particle size distribution and standard deviation (SD) of Ag nanoparticles prepared by the arc plasma method with arc currents of (a) 40, (b) 50, and (c) $80 \mathrm{~A}$. 


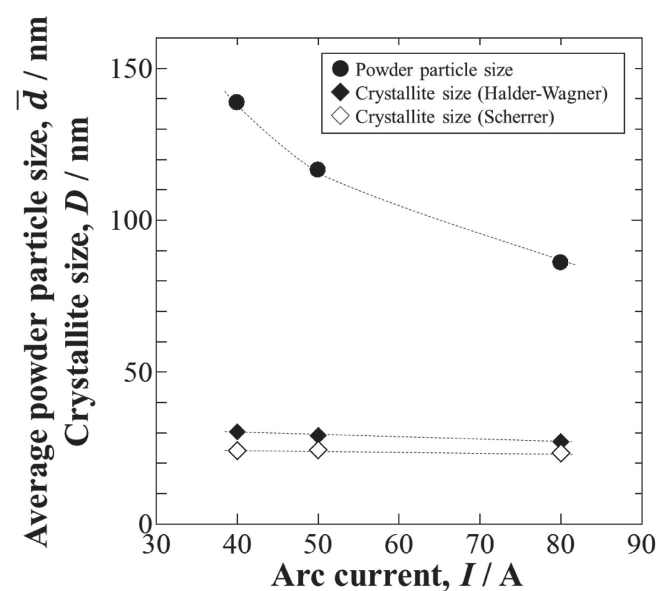

Fig. 6 Average powder particle size $\bar{d}$ and the crystallite size $D$ of each Ag nanoparticle sample.

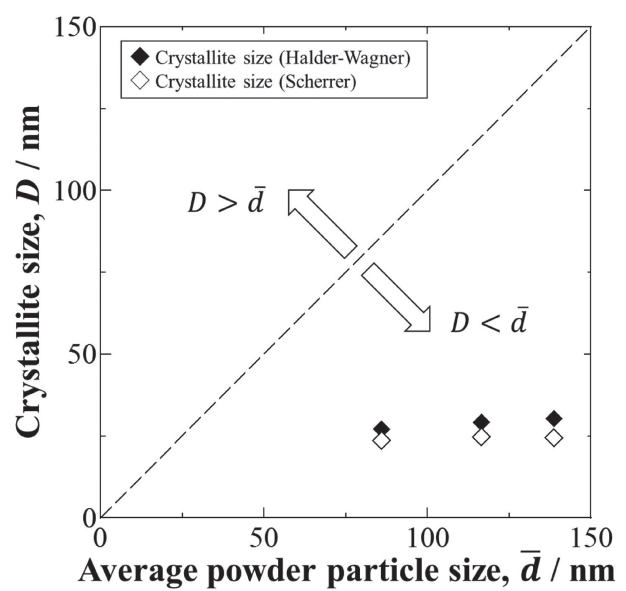

Fig. 7 Relationship between the crystallite size $D$ and the average powder particle size $\bar{d}$ of Ag nanoparticles prepared by the arc plasma method under various conditions.

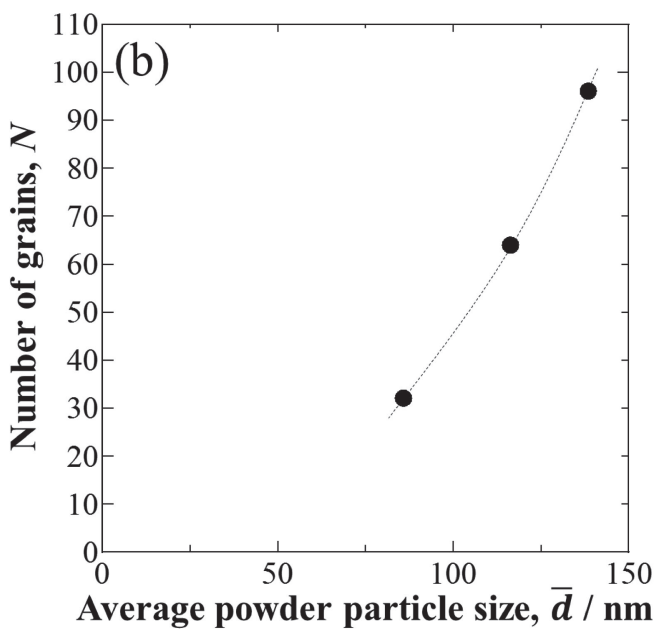

Fig. 8 Number of grains in each Ag nanoparticle as a function of (a) the arc current and (b) the average powder particle size $\bar{d}$.

current and gas atmosphere. The average Ag particle size in this study was smaller than that in previous studies (highpressure water atomization ${ }^{7)}$ and chemical reduction process$\mathrm{es}^{8)}$ ). Therefore, it is concluded that small, uniform, and spherical Ag nanoparticles can be prepared by the arc plasma method.

As shown in Fig. 6, the crystallite size $D$ was smaller than the average particle size $\bar{d}$, regardless of the condition of the arc plasma method. Figure 7 shows the relationship between the crystallite size $D$ and the average particle size $\bar{d}$ of each sample. The dashed line in Fig. 7 means $D=\bar{d}$. Consequently, it is expected that Ag nanoparticles are polycrystal if the data point is below the dashed line $(D<\bar{d})$. The data points of all Ag nanoparticles in this study were below the dashed line; therefore, it is concluded that they were polycrystalline particles. Here, the average volume of $\mathrm{Ag}$ nanoparticles evaluated using the average particle size $\bar{d}$ is expressed as $V$. The number of grains $N$ contained in each spherical Ag nanoparticle is represented by the following formula:

$$
N=\frac{V}{4 \pi(D / 2)^{3} / 3} .
$$

Here, it was simply assumed that the Ag nanoparticles and grains of crystallites were spherical. In eq. (4), the crystallite size $D$ evaluated by the Halder-Wagner method is used for the calculation (Fig. 6). Figure 8(a) shows $N$ as a function of the arc current, and Fig. 8(b) shows the relationship between the average particle size $\bar{d}$ and $N$. It was found that $N$ decreased with increasing arc current and decreasing average particle size $\bar{d}$ because the arc current dependence of the crystallite size is lower than that of the particle size. The use of polycrystalline nanoparticles contributes to the grain refinement of sintered materials which are made with them. Therefore, the findings of this study indicate that $\mathrm{Ag}$ nanoparticles can be applied as a raw material for small electronic parts and for high-strength Ag sintered materials having very fine grains.

\section{Conclusions}

In this study, Ag nanoparticles were prepared by the arc plasma method and their properties were investigated.

(1) It was confirmed that the prepared Ag nanoparticles consisted of a single FCC Ag phase by XRD analysis. Moreover, no $\mathrm{Ag}$ oxides were observed in the 
nanoparticles by either FE-SEM observations or XRD analysis.

(2) The average particle size of the Ag nanoparticles prepared by the arc plasma method with the arc current of $80 \mathrm{~A}$ was $86.0 \mathrm{~nm}$. The shape of the $\mathrm{Ag}$ nanoparticle was spherical.

(3) It was found that the particle size distribution of $\mathrm{Ag}$ nanoparticles depends on the arc current. An increase in the arc current contributes to decrease the average particle size and SD of the particle size distribution. The arc current dependence of the crystallite size is lower than that of the particle size. Moreover, the crystallite size was smaller than the average particle size, regardless of the arc current. Consequently, it is concluded that all of the Ag nanoparticles prepared in this study were polycrystalline.

\section{Acknowledgments}

The authors would like to thank Mr. Y. Kamite of Kamitec Corporation and Mr. T. Sekine of Akita Industrial Technology Center for experimental support and valuable discussions.

\section{REFERENCES}

1) T. Mineta and H. Sato: Mater. Sci. Eng. A 735 (2018) 418-422.

2) G. Gille, B. Szesny, K. Dreyer, H. van den Berg, J. Schmidt, T. Gestrich and G. Leitner: Int. J. Refract. Met. Hard Mater. 20 (2002) 3-22.

3) L.J. Kai, L.Y. Hung, L.W. Wu, M.Y. Chiang, D.S. Jiang, C.M. Huang and Y.P. Wang: Electronic Components and Technology Conference, (2012) pp. 1163-1168.

4) G.C. Cho, L.M. Kaneko, T.E. Donovan and S.N. White: J. Prosthet. Dent. 82 (1999) 272-276.

5) G.R. Khayati and K. Janghorban: Adv. Powder Technol. 23 (2012)
393-397.

6) A.T. Ankus and R.D. Venter: Powder Technol. 73 (1992) 169-179.

7) T. Ogihara, T. Kubo, K. Minami, H. Yasuda, Y. Sato, N. Aoyagi, S Arita, M. Harada and A. Harada: J. Jpn. Soc. Powder Metallurgy 63 (2016) 1027-1031.

8) Z. Zhang, B. Zhao and L. Hu: J. Solid State Chem. 121 (1996) 105110.

9) S. Panda, J.-J. Fundenberger, Y. Zhao, J. Zou, L.S. Toth and T. Grosdidier: Int. J. Hydrogen Energy 42 (2017) 22438-22448.

10) Z.Q. Wei, T.D. Xia, L.F. Bai, J. Wang, Z. Wu and P. Yan: Mater. Lett. 60 (2006) 766-770.

11) Z. Wei, H. Qiao, H. Yang, C. Zhang and X. Yan: J. Alloys Compd. 479 (2009) 855-858.

12) K.S. Chou and C.Y. Ren: Mater. Chem. Phys. 64 (2000) 241-246.

13) A.T. Ankus and R.D. Venter: Powder Technol. 73 (1992) 169-179.

14) T.C. Pluym, Q.H. Powell, A.S. Gurav, T.L. Ward, T.T. Kodas, L.M. Wang and H.D. Glicksman: J. Aerosol Sci. 24 (1993) 383-392.

15) G.R. Khayati and K. Janghorban: Adv. Powder Technol. 23 (2012) 393-397.

16) M. Zhou, Z. Wei, H. Qiao, L. Zhu, H. Yang and T. Xia: J. Nanomater. 2009 (2009) 1-5.

17) T. Uchikoshi, Y. Sakka and E. Ozawa: J. Japan Inst. Metals 53 (1989) 614-620.

18) Y. Sakka, T. Uchikoshi and E. Ozawa: J. Japan Inst. Metals 53 (1989) $422-428$.

19) M. Farbod, A. Movahed and I. Kazeminezhad: Mater. Lett. 89 (2012) $140-142$.

20) A.R. Mohammadian, S. Hajarpour, Kh. Gheisari and M. Farbod: Mater. Lett. 133 (2014) 91-93.

21) M. Farbod, V. Kargar dehbidi and M.Z. Shoushtari: Ceram. Int. 43 (2017) 13670-13676.

22) N.C. Halder and C.N.J. Wagner: Advances in X-Ray Analysis 9 (1966) 91-102.

23) P. Scherrer: Mathematisch-Physikalische Klasse 2 (1918) 98-100.

24) The Japan Institute of Metals and Materials: Kinzoku Data Book 4th ed., (Maruzen, Japan, 2004).

25) Y. Yue, D. Yuchi, P. Guan, J. Xu, L. Guo and J. Liu: Nat. Commun. 7 (2016) 12251-12257.

26) J.K. Baird, T.R. King and C. Stein: J. Phys. Chem. Solids 60 (1999) 891-894. 